\title{
INVARIANT SUBSPACES AND PERTURBATIONS
}

\author{
LYLE NOAKES
}

(Communicated by Palle E. T. Jorgensen)

\begin{abstract}
We study the stability of proper closed invariant subspaces with respect to perturbations in norm of continuous operators on a Hilbert space, using a nonlinear $C^{\infty}$ map of Banach spaces.
\end{abstract}

\section{INTRODUCTION}

When $J$ and $K$ are Banach spaces over $\mathbb{C}$ or $\mathbb{R}$, let $L(J, K)$ be the Banach space of continuous linear transformations from $J$ to $K$, with the operator norm. Let $(H,<,>)$ be a Hilbert space; a subspace $W$ of $H$ is proper when $W \neq\{\mathbf{0}\}, H$. Let $P(H)$ be the closed subset of $L(H, H)$ consisting of the orthogonal projections $p_{W}$ onto closed subspaces $W$ of $H$, and let $W^{\perp}$ denote the orthogonal complement of $W$ in $H$. If $W$ is a closed subspace of $H$, identify $L(W, K)$ with $\left\{A \in L(H, K): A \mid W^{\perp}=\mathbf{0}\right\}$.

Let $A_{0} \in L(H, H)$ and $W_{0}$ be a closed subspace of $H: W_{0}$ is invariant for $A_{0}$ when $A_{0}\left(W_{0}\right) \subseteq W_{0}$. Let $W_{0}$ be proper. In $\S 2 U=B(\mathbf{0}, 1) \cap L\left(W_{0}, W_{0}^{\perp}\right)$, and we define a $C^{\infty}$ function $\Psi_{W_{0}}: L(H, H) \times U \rightarrow L\left(W_{0}, W_{0}^{\perp}\right)$ with the property that $\Psi_{W_{0}}(A, T)=\mathbf{0} \Leftrightarrow(\mathbf{1}+T) W_{0}$ is an invariant subspace for $A$. Then we compute the derivative of $\Psi_{W_{0}}$.

In $\S 3 W_{0}$ is a proper closed invariant subspace for $A_{0}$, and we define stability of $\left(W_{0}, A_{0}\right)$ in $\mathscr{I}$ relative to a smaller closed invariant subspace $V_{0} \subseteq W_{0}$ of $A_{0}$. Here $\mathscr{I}$ is closed ideal in $L(H, H)$. Unique stability is a stronger condition.

Let $W_{1}$ be the orthogonal complement of $V_{0}$ in $W_{0}$. In the definition of stability, $A_{0}$ is perturbed by elements of $\mathscr{F}$ which vanish on $V_{0}$, and we are concerned with invariant subspaces of the form $(1+T) W_{0}$ where $T \in$ $\mathscr{I} \cap L\left(W_{1}, W_{0}^{\perp}\right)$. So no nontrivial perturbation is allowed on $V_{0}$, but in some applications $V_{0}=\{\mathbf{0}\}$. The case $\mathscr{I}=L(H, H)$ also occurs in applications. If (say) $\mathscr{I}$ is the ideal of compact operators then, roughly speaking, the perturbations of $A_{0}$ and $W_{0}$ are compact.

In $\S 4$ the calculation of $\left(d \Psi_{A_{0}}\right)_{\left(A_{0}, 0\right)}$ leads to a necessary condition for $\left(W_{0}, A_{0}\right)$ to be stable. For example, take $V_{0}=\{\boldsymbol{0}\}, \mathscr{I}=L(H, H)$, and let $H$

Received by the editors January 21, 1990.

1980 Mathematics Subject Classification (1985 Revision). Primary 47A15.

Key words and phrases. Hilbert space, Banach space, invariant subspace, implicit function theorem. 
be separable. If $A_{0}$ is compact and both $W_{0}$ and $W_{0}^{\perp}$ are infinite-dimensional, then $\left(W_{0}, A_{0}\right)$ is not stable.

In $\S 5$ we give a sufficient condition for $\left(W_{0}, A_{0}\right)$ to be uniquely stable, and this is sometimes satisfied when the spectrum of $A_{0}$ is connected: then a perturbation of $A_{0}$ also has a proper closed invariant subspace. When the spectrum of $A_{0}$ is disconnected, our stability result does not seem to follow easily from the Riesz decomposition.

\section{Projections AND DeRIVATIVES}

Let $A_{0} \in L(H, H)$, and let $W_{0}$ be a proper closed subspace of $H$. Define $W \subseteq H$ and $\Psi_{A_{0}, W_{0}}: U \equiv B(\mathbf{0}, 1) \cap L\left(W_{0}, W_{0}^{\perp}\right) \rightarrow L\left(W_{0}, W_{0}^{\perp}\right)$ by $W=$ $(\mathbf{1}+T) W_{0}$ and $\Psi_{A_{0}, W_{0}}(T)=\left(\mathbf{1}-p_{W_{0}}\right) \circ\left(\mathbf{1}-p_{W}\right) \circ A_{0} \circ(\mathbf{1}+T) \mid W_{0}$. Because $T \in U, \mathbf{1}+T$ is invertible.

If $w \in W_{0} \cap W^{\perp}$ then $\mathbf{0}=\langle w,(\mathbf{1}+T) w\rangle=\langle w, w\rangle$, and therefore, $W_{0} \cap$ $W^{\perp}=\{\mathbf{0}\}$. If $\left(\mathbf{1}-p_{W_{0}}\right)\left(\mathbf{1}-p_{W}\right)(u)=\mathbf{0}$ then $\left(\mathbf{1}-p_{W}\right)(u) \in W_{0} \Rightarrow\left(\mathbf{1}-p_{W}\right)(u) \in$ $W_{0} \cap W^{\perp}=\{\mathbf{0}\}$. Therefore $\operatorname{Ker}\left(\mathbf{1}-p_{W_{0}}\right) \cap \operatorname{Im}\left(\mathbf{1}-p_{W}\right)=\{\mathbf{0}\}$. So $W$ is an invariant subspace for $A_{0} \Leftrightarrow \Psi_{A_{0}, W_{0}}(T)=0$.

Lemma 1. The assignment $T \mapsto p_{W}$ is $C^{\infty}$ in the sense of [1], and $\left(d p_{W}\right)_{T}$ is given by $\left(d p_{W}\right)_{T}(D) \mid W=\left(\mathbf{1}-p_{W}\right) \circ D \circ(\mathbf{1}+T)^{-1}$ and $\left(d p_{W}\right)_{T}(D) \mid W^{\perp}=$ $\left(\mathbf{1}+T^{*}\right)^{-1} \circ D^{*} \circ\left(\mathbf{1}-p_{W}\right)^{*}$ where $D \in L\left(W_{0}, W_{0}^{\perp}\right)$.

Proof. Define a $C^{\infty}$ function $\mathscr{F}: U \times L(H, H) \rightarrow L\left(W_{0}, H\right) \oplus L\left(W_{0}^{\perp}, H\right)$ by $\mathscr{F}(T, p)=\left((\mathbf{1}-p) \circ(\mathbf{1}+T)\left|W_{0}, \quad p \circ\left(\mathbf{1}+T^{*}\right)^{-1}\right| W_{0}^{\perp}\right)$. Then $\mathscr{F}(T, p)=$ $\mathbf{0} \Leftrightarrow p=p_{W}$ where $W=(\mathbf{1}+T) W_{0}$. If $\mathscr{F}(T, p)=\mathbf{0}$, then $(d \mathscr{F})_{T, p}(0, q)=$ $\left(-q \circ(\mathbf{1}+T)\left|W_{0}, q \circ\left(\mathbf{1}+T^{*}\right)^{-1}\right| W_{0}^{\perp}\right)$, and $T \mapsto p_{W}$ is $C^{\infty}$ by the implicit function theorem [1].

Because $\left(\mathbf{1},-p_{W}\right) \circ(\mathbf{1}+T)\left|W_{0}=0,\left(d p_{W}\right)_{T}(D) \circ(\mathbf{1}+T)\right| W_{0}=\left(\mathbf{1}-p_{W}\right) \circ$ $D \mid W_{0}$, or rather $\left(d p_{W}\right)_{T}(D) \mid W=\left(1-p_{W}\right) \circ D \circ(1+T)^{-1}$. Now $p_{W} \circ p_{W}=p_{W}$ and therefore, $\left(d p_{W}\right)_{T}(D) \circ p_{W}+p_{W} \circ\left(d p_{W}\right)_{T}(D)=\left(d p_{W}\right)_{T}(D)$ so that, if $v \in$ $W^{\perp}, p_{W} \circ\left(d p_{W}\right)_{T}(D)(v)=\mathbf{0}$; namely, $\left(d p_{W}\right)_{T}^{\prime}(D)\left(W^{\perp}\right) \subseteq W$.

Now $\left\langle p_{W} u_{1}, u_{2}\right\rangle=\left\langle u_{1}, p_{W} u_{2}\right\rangle$ for all $u_{1}, u_{2} \in H$, so $\left\langle\left(d p_{W}\right)_{T}(D) u_{1}, u_{2}\right\rangle=$ $\left\langle u_{1},\left(d p_{W}\right)_{T}(D) u_{2}\right\rangle$. In particular, when $u_{1}=v \in W^{\perp}$ and $u_{2}=w \in W$, $\left\langle\left(d p_{W}\right)_{T}(D) v, w\right\rangle=\left\langle v,\left(d p_{W}\right)_{T}(D) w\right\rangle=\left\langle v,\left(\mathbf{1}-p_{W}\right) \circ D \circ(\mathbf{1}+T)^{-1} w\right\rangle=$ $\left\langle\left(1+T^{*}\right)^{-1} \circ D^{*} \circ\left(1-p_{W}\right)^{*} v, w\right\rangle$. This proves Lemma 1 .

Define a $C^{\infty}$ function $\Psi_{W_{0}}: L(H, H) \times U \rightarrow L\left(W_{0}, W_{0}^{\perp}\right)$, by $\Psi_{W_{0}}(A)=$ $\Psi_{A, W_{0}}$. Then $\Psi_{W_{0}}(A, T)=\left(\mathbf{1}-p_{W_{0}}\right) \circ\left(\mathbf{1}-p_{W}\right) \circ A \circ(\mathbf{1}+T) \mid W_{0}$ is the composite of

(i) inclusion of $W_{0}$ in $H$ (independent of $T$ ),

(ii) $(\mathbf{1}+T): H \rightarrow H$,

(iii) $A \mid H: H \rightarrow H$ (independent of $T$ ),

(iv) $1-p_{W}: H \rightarrow H$.

(v) $1-p_{W_{0}}: H \rightarrow H$ (independent of $T$ ).

Therefore $\left(d \Psi_{W_{0}}\right)_{(A, T)}(\mathbf{0}, D) \in L\left(W_{0}, W_{0}^{\perp}\right)$ is $\left(\mathbf{1}-p_{W_{0}}\right) \circ\left\{\left(\mathbf{1}-p_{W}\right) \circ A \circ D-\right.$ $\left.\left.\left(d p_{W}\right)_{T}(D) \circ A \circ(\mathbf{1}+T) \mid W_{0}\right)\right\}$, which we rewrite as $\left(\mathbf{1}-p_{W_{0}}\right) p_{W} \circ\left\{\left(\mathbf{1}-p_{W}\right) \circ A \circ D\right.$ $\left.-\left(d p_{W}\right)_{T}(D) \circ A \circ(\mathbf{1}+T)\left|W_{0}-\left(d p_{W}\right)_{T}(D) \circ\left(\mathbf{1}-p_{W}\right) \circ A \circ(\mathbf{1}+T)\right| W_{0}\right\}$. From Lemma 1 we now obtain 


\section{Lemma 2.}

$$
\begin{aligned}
&\left(d \Psi_{W_{0}}\right)_{(A, T)}(\mathbf{0}, D) \\
&=\left(\mathbf{1}-p_{W_{0}}\right) \circ\left\{\left(\mathbf{1}-p_{W}\right) \circ A \circ D-\left\{\left(\mathbf{1}-p_{W}\right) \circ D \circ(\mathbf{1}+T)^{-1} \circ p_{W}\right.\right. \\
&\left.\left.+\left(\mathbf{1}+T^{*}\right)^{-1} \circ D^{*} \circ\left(\mathbf{1}-p_{W}\right)^{*} \circ\left(\mathbf{1}-p_{W}\right)\right\} \circ A \circ(\mathbf{1}+T) \mid W_{0}\right\} .
\end{aligned}
$$

In particular,

$$
\begin{aligned}
& \left(d \Psi_{W_{0}}\right)_{(A, \mathbf{0})}(\mathbf{0}, D) \\
& \quad=\left(\mathbf{1}-p_{W_{0}}\right) \circ\left\{A \circ D-D \circ p_{W_{0}} \circ A\left|W_{0}-D^{*} \circ\left(\mathbf{1}-p_{W_{0}}\right)^{*} \circ\left(\mathbf{1}-p_{W_{0}}\right) \circ A\right| W_{0}\right\} .
\end{aligned}
$$

Suppose now that $W_{0}$ is an invariant subspace for $A_{0} \in L(H, H)$. Then $\left(\mathbf{1}-p_{W_{0}}\right) \circ A_{0} \mid W_{0}=\mathbf{0}$, and we have

\section{Lemma 3.}

$$
\left.\left(d_{\Psi_{W_{0}}}\right)_{\left(A_{0}, \mathbf{0}\right)}(\mathbf{0}, D), D\right)=\Lambda\left(W_{0}, A_{0}\right)(D) \equiv\left(\mathbf{1}-p_{W_{0}}\right)\left\{A_{0} \circ D-D \circ p_{W_{0}} \circ A_{0} \mid W_{0}\right\}
$$

\section{Stability in AN IDEAL RElative to A ClOSED INVARIANT SUBSPACE}

Let $W_{0}$ be a closed subspace of $H$, and let $\mathscr{I}$ be a closed two-sided ideal of the algebra $L(H, H)$. (Note that $H$ is not necessarily separable, so that $\mathscr{J}$ is not necessarily the ideal of compact operators.)

Lemma 4. For some $\varepsilon \in(0,1]$, there is a $C^{\infty}$ function $\delta: U_{\varepsilon} \equiv B(\mathbf{0}, \varepsilon) \cap \mathscr{J} \rightarrow$ $\mathscr{I}$, such that $\delta(T)=p_{W}-p_{W_{0}}$ for all $T \in U_{\varepsilon}$. Here $W=(1+T) W_{0}$.

Proof. Define a $C^{\infty}$ function $\mathscr{G}: U_{1} \times \mathscr{I} \rightarrow \mathscr{I} \cap L\left(W_{0}, H\right) \oplus \mathscr{I} \cap L\left(W_{0}^{\perp}, H\right)$ by

$$
\mathscr{G}(T, d)=\left(\left(\mathbf{1}-p_{W_{0}}-d\right) \circ(\mathbf{1}+T)\left|W_{0}, \quad\left(p_{W_{0}}+d\right) \circ\left(\mathbf{1}+T^{*}\right)^{-1}\right| W_{0}^{\perp}\right) .
$$

Note that $\mathscr{G}$ maps into $\mathscr{I} \times \mathscr{I}$ because $\mathscr{I}$ is a two-sided ideal, and that $\mathscr{G}(T, d)=\mathbf{0} \Leftrightarrow p_{W_{0}}+d=p_{W}$. If $\mathscr{G}(T, d)=\mathbf{0}$ then $(d \mathscr{G})_{T, d}(\mathbf{0}, e)=$ $\left(-e \circ(\mathbf{1}+T)\left|W_{0}, e \circ\left(\mathbf{1}+T^{*}\right)^{-1}\right| W_{0}^{\perp}\right)$. Therefore, by the implicit function theorem, for some $\varepsilon$, there is a $C^{\infty}$ function $\delta$ such that $\mathscr{G}(T, \delta(T))=\mathbf{0}$ for all $T \in U_{\varepsilon}$. This proves Lemma 4 .

Now suppose that $W_{0}$ is a proper closed invariant subspace for $A_{0} \in L(H, H)$, and let $V_{0}$ be a closed subspace of $W_{0}$ (not necessarily proper), which is also invariant for $A_{0}$. Write $W_{0}=V_{0} \oplus W_{1}$. Let $\mathscr{J}_{W_{0}, V_{0}, A_{0}}$ be the closed affine subspace $\left\{A_{0}+S: S \in \mathscr{I}, S \mid V_{0}=\mathbf{0}\right\}$ of $L(H, H)$.

We say that $\left(W_{0}, A_{0}\right)$ is stable in $\mathscr{I}$ relative to $V_{0}$, when there is an open neighborhood $N_{0}$ of $A_{0}$ in $\mathscr{I}_{W_{0}, V_{0}, A_{0}}$ and a $C^{1}$ function $f: N_{0} \rightarrow \mathscr{I} \cap$ $L\left(W_{1}, W_{0}^{\perp}\right)$, such that $f\left(A_{0}\right)=\mathbf{0}$, and $(\mathbf{1}+f(A)) W_{0}$ is an invariant subspace for $A$, for each $A \in N_{0}$. When $N_{0}$ can be chosen so that $f$ is unique we call $\left(W_{0}, A_{0}\right)$ uniquely stable in $\mathscr{I}$ relative to $V_{0}$. Note that if $\mu \in \mathbb{C}$ then $\left(W_{0}, A_{0}\right)$ is (uniquely) stable in $\mathscr{I}$ relative to $V_{0} \Leftrightarrow\left(W_{0}, A_{0}-\mu \mathbf{1}\right)$ is (uniquely) stable in $\mathscr{I}$ relative to $V_{0}$.

If $A \in \mathscr{I}_{W_{0}, V_{0}, A_{0}}, T \in \mathscr{I} \cap L\left(W_{1}, W_{0}^{\perp}\right)$, write $W=(1+T) W_{0}$. Then

$$
\begin{aligned}
\Psi_{A, W_{0}}(T) \equiv & \left(\mathbf{1}-p_{W_{0}}\right) \circ\left(\mathbf{1}-p_{W}\right) \circ A_{0} \circ(\mathbf{1}+T) \mid W_{0} \\
= & \left(\mathbf{1}-p_{W_{0}}\right) \circ A_{0} \circ(\mathbf{1}+T)\left|W_{0}+\left(\mathbf{1}-p_{W_{0}}\right) \circ S \circ(\mathbf{1}+T)\right| W_{0} \\
& -\left(\mathbf{1}-p_{W_{0}}\right) \circ p_{W} \circ\left(A_{0}+S\right) \circ(\mathbf{1}+T) \mid W_{0} \\
= & \left(\mathbf{1}-p_{W_{0}}\right) \circ A_{0} \circ T+\left(\mathbf{1}-p_{W_{0}}\right) \circ S \circ(\mathbf{1}+T) \mid W_{0} \\
& -\left(\mathbf{1}-p_{W_{0}}\right) \circ p_{W} \circ\left(A_{0}+S\right) \circ(\mathbf{1}+T) \mid W_{0}
\end{aligned}
$$


because $W_{0}$ is an invariant subspace for $A_{0}$.

Choose $\varepsilon$ as in Lemma 4, and suppose that $T \in U_{\varepsilon} \cap L\left(W_{1}, W_{0}^{\perp}\right)$. Then $\left(1-p_{W_{0}}\right) \circ p_{W} \in \mathscr{I}$, and therefore $\Psi_{A, W_{0}}(T) \in \mathscr{I} \cap L\left(W_{0}, W_{0}^{\perp}\right)$. If $v_{0} \in V_{0}$ then

$$
\begin{aligned}
\Psi_{A, W_{0}}(T)\left(v_{0}\right) & =\left(\mathbf{1}-p_{W_{0}}\right) \circ\left(\mathbf{1}-p_{W}\right) \circ A\left(v_{0}\right)=\left(\mathbf{1}-p_{W_{0}}\right) \circ\left(\mathbf{1}-p_{W}\right) \circ A_{0}\left(v_{0}\right) \\
& =\left(\mathbf{1}-p_{W_{0}}\right) \circ\left(\mathbf{1}-p_{W}\right) \circ(\mathbf{1}+T) \circ A_{0}\left(v_{0}\right)=\mathbf{0}
\end{aligned}
$$

because $T\left(v_{0}\right)=\mathbf{0}, S\left(v_{0}\right)=\mathbf{0}, A_{0}\left(v_{0}\right) \in W_{0}$, and $T \circ A_{0}\left(v_{0}\right)=\mathbf{0}$. This, together with Lemma 3, proves

Lemma 5. $\Psi_{W_{0}}$ restricts to a $C^{\infty}$ map

$$
\Psi_{W_{0}}^{\prime}: \mathscr{I}_{W_{0}, V_{0}, A_{0}} \times\left\{U_{\varepsilon} \cap L\left(W_{1}, W_{0}^{\perp}\right)\right\} \rightarrow \mathscr{I} \cap L\left(W_{1}, W_{0}^{\perp}\right)
$$

and

$$
\begin{array}{r}
\left(d \Psi_{W_{0}}^{\prime}\right)_{\left(A_{0}, \mathbf{0}\right)}(\mathbf{0}, D)=\Lambda\left(W_{0}, A_{0}\right)(D) \in \mathscr{I} \cap L\left(W_{1}, W_{0}^{\perp}\right), \\
\text { where } D \in \mathscr{I} \cap L\left(W_{1}, W_{0}^{\perp}\right) .
\end{array}
$$

\section{A NECESSARY CONDITION FOR STABILITY}

In the setting of $\S 3$ we have

Theorem 1. If $\left(W_{0}, A_{0}\right)$ is stable in $\mathscr{I}$ relative to $V_{0}$, then $\Lambda\left(W_{0}, A_{0}\right) \mid \mathscr{I} \cap$ $L\left(W_{1}, W_{0}^{\perp}\right)$ maps onto the whole of $\mathscr{I} \cap L\left(W_{1}, W_{0}^{\perp}\right)$.

Proof. Suppose that $\left(W_{0}, A_{0}\right)$ is stable in $\mathscr{I}$ relative to $V_{0}$. For $A \in$ $\mathrm{f}^{-1}\left\{U_{\varepsilon} \cap L\left(W_{1}, W_{0}^{\perp}\right)\right\}, \Psi_{W_{0}}^{\prime}(A, f(A))=\mathbf{0}$. For $B \in \mathscr{I} \cap L\left(W_{1}, W_{0}^{\perp}\right)$,

$$
\left(d \Psi_{W_{0}}^{\prime}\right)_{\left(A_{0}, \mathbf{0}\right)} \circ\left(\mathbf{0},(d f)_{A_{0}}(B)\right)+\left(d \Psi_{W_{0}}^{\prime}\right)_{\left(A_{0}, \mathbf{0}\right)}(B, \mathbf{0})=\mathbf{0} .
$$

Now $\left(d \Psi_{W_{0}}^{\prime}\right)_{\left(A_{0}, \mathbf{0}\right)}(\mathbf{0}, D)=\Lambda\left(W_{0}, A_{0}\right)(D)$, and $\Lambda\left(W_{0}, A_{0}\right) \circ(d f)_{A_{0}}(B)=$ $-\left(d \Psi_{W_{0}}^{\prime}\right)_{\left(A_{0}, \mathbf{0}\right)}(B, \mathbf{0})=-\Psi_{W_{0}}^{\prime}(B, \mathbf{0})=-\Psi_{W_{0}}(B, \mathbf{0})$, since $\Psi_{W_{0}}^{\prime}(A, T)$ is linear in the variable $A$. Let $\rho: \mathscr{I} \cap L\left(W_{1}, W_{0}^{\perp}\right) \rightarrow L(H, H)$ be the inclusion. Then $\Psi_{W_{0}}(\rho(T), \mathbf{0})=T$ for all $T \in \mathscr{I} \cap L\left(W_{1}, W_{0}^{\perp}\right)$. Therefore $\Lambda\left(W_{0}, A_{0}\right) \circ \chi=$ $\mathbf{1}_{\mathscr{I} \cap L\left(W_{1}, W_{0}^{\perp}\right)}$ where $\chi=-(d \mathrm{f})_{A_{0}} \circ \rho$.

Remark. $\Lambda\left(W_{0}, A_{0}\right) \mid \mathcal{I} \cap L\left(W_{0}, W_{0}^{\perp}\right)$ has nontrivial kernel $\Leftrightarrow A_{0}$ commutes with some $D \in \mathscr{I}$ for which $W_{0}$ is not an invariant subspace.

Example 1. Let $A_{0}=\mathbf{0}$. Then $\Lambda\left(W_{0}, A_{0}\right) \mid \mathscr{I} \cap L\left(W_{1}, W_{0}^{\perp}\right)$ is trivial, and therefore $\left(W_{0}, \mathbf{0}\right)$ is not stable in $\mathscr{I}$ relative to $V_{0}$ unless $\mathscr{I} \cap L\left(W_{1}, W_{0}^{\perp}\right)$ is also trivial. Let $V_{0}=\{\mathbf{0}\}$.

Then there is no $C^{1}$ assignment of proper closed invariant subspaces to operators in a neighborhood of $\mathbf{0}$ in $\mathscr{I}$. The $C^{1}$ assignment would be by continuous isomorphisms, which are perturbations of 1 by elements of $\mathscr{I}$.

We have in mind the case where $H$ is infinite-dimensional, and $\mathscr{I}$ is $L(H, H)$, or perhaps the ideal of compact operators in $L(H, H)$, but when $H=\mathbb{R}^{2}$, with the Euclidean inner product, note the following alternative proof that $\left(\mathbf{0}, W_{0}\right)$ is not stable in $L(H, H)$ relative to $\{0\}$.

Without loss of generality, $W_{0}=\mathbb{R} \times\{\mathbf{0}\}$. Suppose that, for some $\beta>0$, and some continuous $\mathrm{f}: B(\mathbf{0}, \beta) \rightarrow L\left(\mathbb{R}^{2}, \mathbb{R}^{2}\right)$, we have $f(\mathbf{0})=\mathbf{0}$, and $(\mathbf{1}+f(A)) W_{0}$ is an invariant subspace of $A$ whenever $A \in B(\mathbf{0}, \beta)$. 
Let $S^{1}$ be the unit circle in $\mathbb{R}^{2} \cong \mathbb{C}$. For $z \in S^{1}$, let $R_{z} \in L\left(\mathbb{R}^{2}, \mathbb{R}^{2}\right)$ be a complex multiplication by $z$. Define $g(z) \in B(\mathbf{0}, \beta)$ to be

$$
R_{z} \circ\left[\begin{array}{cc}
0 & \beta / 2 \\
0 & 0
\end{array}\right] \circ R_{z}^{-1} \text {. }
$$

The only one-dimensional invariant subspace for $g(z)$ is $R_{z} W_{0}$ and therefore, $(1+f \circ g(z)) W_{0}=R_{z} W_{0}$.

Define $f: B(\mathbf{0}, \beta) \rightarrow \mathbb{R} P^{1}$ by $f(A)=(1+f(A)) W_{0}$, and $R: S^{1} \rightarrow \mathbb{R} P^{1}$ by $R(z)=R_{z} W_{0}$. Then $R=f \circ g: S^{1} \rightarrow B(\mathbf{1}, \beta) \rightarrow \mathbb{R} P^{1}$, which is a contradiction, since $R$ is the double cover, and $B(\mathbf{0}, \beta)$ is contractible.

Example 2. Suppose $A_{0} \in \mathcal{J}$ where $\mathcal{J}$ is a two-sided idea of $L(H, H)$ and $\mathscr{J} \cap L\left(W_{1}, W_{0}^{\perp}\right) \neq \mathscr{I} \cap L\left(W_{1}, W_{0}^{\perp}\right)$. Then $\Lambda\left(W_{0}, A_{0}\right)\left\{\mathscr{I} \cap L\left(W_{1}, W_{0}^{\perp}\right)\right\} \neq$ $\mathscr{I} \cap L\left(W_{1}, W_{0}^{\perp}\right)$, and therefore $\left(W_{0}, A_{0}\right)$ is not stable in $\mathscr{I}$ relative to $V_{0}$. For instance, let $H$ be separable, and let $\mathcal{J}$ be the ideal of compact transformations in $L(H, H)$. Suppose neither $W_{0}$ nor $W_{0}^{\perp}$ is finite dimensional: then $W_{0} \cong$ $W_{0}^{\perp} \cong L^{2}[0,1]$, and therefore, $\mathscr{I} \cap L\left(W_{0}, W_{0}^{\perp}\right) \neq L\left(W_{0}, W_{0}^{\perp}\right)$. So $\left(W_{0}, A_{0}\right)$ is not stable in $L(H, H)$ relative to $\{0\}$.

\section{A SUFFICIENT CONDITION FOR UNIQUE STABILITY}

Still in the setting of $\S 3$, we have

Lemma 6. If $\Lambda\left(W_{0}, A_{0}\right) \mid \mathscr{I} \cap L\left(W_{1}, W_{0}^{\perp}\right): \mathscr{I} \cap L\left(W_{1}, W_{0}^{\perp}\right) \rightarrow \mathscr{I} \cap L\left(W_{1}, W_{0}^{\perp}\right)$ has a continuous inverse then $\left(W_{0}, A_{0}\right)$ is uniquely stable in $\mathscr{J}$ relative to $V_{0}$. Proof. If $\Lambda\left(W_{0}, A_{0}\right) \mid \mathscr{I} \cap L\left(W_{1}, W_{0}^{\perp}\right)$ has a continuous inverse then, by Lemma 5 and the implicit function theorem, there is a unique $C^{\infty}$ function $f$ defined over some neighborhood $N_{0}$ of $A_{0}$ in $\mathscr{J}_{W_{0}, V_{0}, A_{0}}$ with $f\left(A_{0}\right)=\mathbf{0}$ and $\Psi_{W_{0}}^{\prime}(A, f(A))=\mathbf{0}$ for all $A \in f^{-1}\left\{U_{\varepsilon} \cap L\left(W_{1}, W_{0}^{\perp}\right)\right\}$. Then $(\mathbf{1}+f(A)) W_{0}$ is an invariant subspace for $A$

If $A \in L(H, H)$ write

$$
\begin{aligned}
A_{1}=p_{W_{0}} \circ A \mid W_{0}: W_{0} \rightarrow W_{0} ; & A_{2}=p_{W_{0}} \circ A \mid W_{0}^{\perp}: W_{0}^{\perp} \rightarrow W_{0} ; \\
A_{3}=\left(1-p_{W_{0}} \circ A \mid W_{0}: W_{0} \rightarrow W_{0}^{\perp} ;\right. & A_{4}=\left(1-p_{W_{0}}\right) \circ A \mid W_{0}^{\perp}: W_{0}^{\perp} \rightarrow W_{0}^{\perp} .
\end{aligned}
$$

The spectrum of a linear operator $B$ is denoted by $\sigma(B)$.

Theorem 2. If $\sigma\left(p_{W_{1}} \circ A_{0,1} \mid W_{1}\right) \cap \sigma\left(A_{0,4}\right)$ is empty then $\left(W_{0}, A_{0}\right)$ is uniquely stable in $\mathcal{I}$ relative to $V_{0}$.

First, recall Rosenblum's theorem [2, Theorem 0.12]: Let $J, K$ be Hilbert spaces. Given $B \in L(J, J), C \in L(K, K)$, define $B^{*}, C_{*}: L(J, K) \rightarrow$ $L(J, K)$ by $B^{*}(D)=D \circ B$ and $C_{*}(D)=C \circ D$. Then $\sigma\left(C_{*}-B^{*}\right) \subseteq \sigma(C)-$ $\sigma(B)$. It follows that if $M$ is a closed subspace of $L(J, K)$, which is invariant for both $B^{*}$ and $C_{*}$, then $\sigma\left(C_{*}\left|M-B^{*}\right| M\right) \subseteq \sigma(B)$.

To prove Theorem 2, take $J=W_{1}, K=W_{0}^{\perp}, B=p_{W_{1}} \circ A_{0,1} \mid W_{1}, C=$ $A_{0,4}, M=\mathscr{I} \cap L\left(W_{1}, W_{0}^{\perp}\right)$. By hypothesis, $0 \notin \sigma(C)-\sigma(B)$ and, therefore $\Lambda\left(W_{0}, A_{0}\right) \mid \mathscr{J} \cap L\left(W_{1}, W_{0}^{\perp}\right)=C_{*}-B^{*}$ has a continuous inverse. Theorem 2 now follows from Lemma 6. 
Example 3. When $H$ is a complex Hilbert space, a disconnection of $\sigma\left(A_{0}\right)$ leads to the Riesz decomposition of $H$, as a direct sum of invariant subspaces $W_{0}, Y_{0}$ for $A_{0}$ [2, Theorem 2.10]. If $\sigma\left(A_{0}\right)$ is disconnected and $\left\|A-A_{0}\right\|$ is small, then $\sigma(A)$ is also disconnected; therefore $H$ is a direct sum of invariant subspaces $W, Y$ for $A$. A stronger form of stability follows from Theorem 2; namely, $\left(W_{0}, A_{0}\right)$ is uniquely stable in $L(H, H)$ relative to $\{0\}$.

To apply Theorem 2 when $\sigma\left(A_{0}\right)$ is not necessarily disconnected, we take $V_{0} \neq\{\mathbf{0}\}$.

Example 4. Let $A_{0} \in L(H, H)$ be selfadjoint, where $H$ is a complex Hilbert space. Let $\left\{E_{\lambda}: \lambda \in \mathbb{R}\right\} \subseteq P(H)$ be its resolution of the identity: then $A_{0}=$ $\int_{\mathbb{R}} \lambda d E_{\lambda}$. Let $\lambda_{0} \in \sigma\left(A_{0}\right)$. Then $W_{0} \equiv E_{\lambda_{0}}(H)$ is a closed invariant subspace for $A_{0}$.

Suppose that, for some $\alpha>0,\left(\lambda_{0}-\alpha, \lambda_{0}+\alpha\right) \subseteq \sigma\left(A_{0}\right)$. Let $V_{0}$ be the orthogonal complement of $E_{\lambda_{0}-\alpha}(H)$ in $W_{0} \equiv E_{\lambda_{0}}(H)$. By Theorem 2, $\left(W_{0}, A_{0}\right)$ is uniquely stable in $L(H, H)$ relative to $V_{0}$.

For instance, let $H=L^{2}[-1,1]$ and define $A_{0} \in L(H, H)$ by $\left(A_{0}(f)\right) x=$ $x f(x)$ a.e. Let $W_{0}=L^{2}[-1,1 / 2]$ and $V_{0}=L^{2}[1 / 2-\alpha, 1 / 2]$ where $\alpha \in$ $(0,1 / 2)$. Then $\left(W_{0}, A_{0}\right)$ is uniquely stable in $L(H, H)$ relative to $V_{0}$.

In particular, if we perturb $A_{0}$ to a nearly $A \in L\left(L^{2}[-1,1], L^{2}[-1,1]\right)$, which agrees with $A_{0}$ on $L^{2}[1 / 2-\alpha, 1 / 2]$, then $A$ has an invariant subspace of the form $B\left(L^{2}[-1,1 / 2]\right)$ where $B: L^{2}[-1,1] \rightarrow L^{2}[-1,1]$ is continuous linear and close in norm to 1 .

\section{ACKNOWLEDGMENT}

I am especially grateful to Professor Radjavi, whose seminars sparked my interest in the stability problem, and for a number of stimulating conversations. It was also a pleasure to talk to Professors M. Lambrou, W. E. Longstaff, and K. J. Harrison. I want to thank the referee for his careful reading and helpful comments.

\section{REFERENCES}

1. S. Lang, Introduction to differentiable manifolds, Interscience-Wiley, New York 1962.

2. H. Radjavi and P. Rosenthal, Invarint subspaces, Ergeb. Math. (3) Band 77, Springer-Verlag, London 1973.

Department of Mathematics, University of Western Australia, Nedlands, WA 6009, Australia 